\title{
Frame-based stereotaxy in a frameless era: current capabilities, relative role, and the positive- and negative predictive values of blood through the needle
}

\author{
Christopher M. Owen · Mark E. Linskey
}

Received: 29 November 2008/ Accepted: 16 March 2009

(C) The Author(s) 2009. This article is published with open access at Springerlink.com

\begin{abstract}
Introduction In the modern era of frameless stereotaxis (FL), the role of frame-based (FB) stereotactic needle biopsy is evolving. Methods Retrospective review of prospective database of 106 lesions in 91 consecutive patients undergoing FB stereotactic needle biopsy with a systematic "geologic core" technique by a single surgeon. Diagnostic accuracy was calculated comparing biopsy diagnosis with final pathology in 11 patients who underwent subsequent surgical resection. All instances of intra-operative bleeding through the needle were prospectively noted and compared with post-biopsy CT scan. Lesions were classified as risky for FL technique if they were (1) infratentorial or pineal, (2) within $10 \mathrm{~mm}$ of the circle of Willis or root of the Sylvian fissure, or (3) within $10 \mathrm{~mm}$ of deep cerebral veins. Results Diagnostic yield was 94\%. Diagnostic accuracy was $91 \%$. Of 18 lesions involving the corpus callosum, 13 (72.2\%) were GBM 2 were anaplastic astrocytoma, and 1 each were found to be anaplastic oligodendroglioma, primary central nervous system lymphoma (PCNSL) and tumescent MS. Of 25 multifocal lesions, malignant primary brain tumor was diagnosed in 17 (68\%) (11 GBM, 3 PCNSL, 2 anaplastic ologodendroglioma, and 1 anaplastic astrocytoma). Mortality was $0 \%$. Three patients developed temporary neurologic deficits and one had permanent deficit. Absence of persistent blood through the biopsy needle had a negative predicative value of $98.8 \%$ for subsequent neuroimaging blood $>5 \mathrm{~mm}$ diameter. According to our criteria, $80 \%$ of patients would have been candidates for FL biopsy. Conclusions
\end{abstract}

C. M. Owen · M. E. Linskey $(\bowtie)$

Department of Neurological Surgery, University of California,

101 The City Drive South, Bldg. 56, Ste. 400, ZOT 5397, Irvine,

Orange, CA 92868-3298, USA

e-mail: mlinskey@uci.edu
Stereotactic biopsy is an effective, safe and important technique for histologic diagnosis of brain lesions, particularly for multifocal and corpus callosum lesions. Postbiopsy CT can be safely reserved for patients who demonstrate persistent bleeding through the biopsy needle. FB stereotaxy remains an important technique for the $20 \%$ with small or deep seated lesions or when it is advantageous to avoid an incision, a burr hole or general anesthesia.

Keywords Biopsy, needle $\cdot$ Brain neoplasm $\cdot$ Diagnosis · Diagnosis, differential $\cdot$ Neuronavigation .

Stereotaxic techniques

\section{Introduction}

Frame-based stereotaxis (FB) is a well-established technique for performing three-dimensional point stereotactic needle procedures such as diagnostic biopsies, lesion aspirations, and brachytherapy instillation. It has proved a safe and effective tool since it was first coupled with computed planar imaging nearly three decades ago [1-7]. Despite its history, questions still persist regarding FB, including quantifying the risk of non-diagnosis or sampling error for tumor biopsy, and the actual need for post biopsy neuroimaging. Its popularity has also been limited by patient transport logistical issues as well as general neurosurgeons' relative unfamiliarity or discomfort with frame-based equipment and the calculations involved with defining stereotactic entry points, trajectories and targets.

The development of frameless stereotaxis (FL) has been rapidly embraced by most neurosurgeons to the extent that it is rapidly supplanting FB techniques in general neurosurgical practice. Some early reports have suggested that FL techniques are as good or better than the traditional frame- 
based approach $[8,9]$. Recent large series seem to indicate similar diagnostic yields and complication rates, but have failed to agree upon factors such as differences in cost and operating room time between the two methods $[10,11]$. This suggests surgeon and institution experience and preference as major contributing factors and makes clear the necessity of continued investigation and reporting of clinical experience with these techniques. The percentage of potential cases that can safely and effectively approached using FL versus FB technique, the relative desirability and risk of local versus general anesthesia as well as the need for open burr hole versus twist drill are all areas for ongoing debate. Thus the relative role of both FB and FL in modern neurosurgical practice warrants further study.

\section{Methods}

In the 44 month period from December 12, 1998 through September 2, 2003 (four years, eight months) 106 brain lesions in 91 patients underwent 95 consecutive FB cerebral needle biopsy procedures by a single surgeon (MEL) at one university neurosurgical service consisting of a university hospital, a free-standing specialty pediatric hospital, and a Veteran's Administration (VA) hospital. All patients were entered into, and followed on a prospective clinical outcomes database. Patients with multifocal solid lesions only underwent stereotactic biopsy if they did not have a history of primary-histology-proven malignancy within the previous two years, and then only if systemic work-up with chest-, abdomen- and pelvis-computed tomography (CT), as well as nuclear medicine bone scan failed to reveal systemic disease that could be safely biopsied in lieu of one of the cerebral lesions.

\section{Surgical technique}

Biopsy technique included both the Cosman-Roberts-Wells (CRW) stereotactic system (Integra, Plainsboro, NJ) and the Leksell Model G stereotactic system (Elekta, Inc, Norcross,
GA). Targeting imaging included both magnetic resonance (MR) and computed tomography (CT), with CT technique predominantly utilized at the VA hospital where MR targeting was unavailable. Entry techniques included burr hole as well as twist drill craniostomy. Entry locations were determined by lesion location and size. Most procedures were performed in awake patients unless age, lesion location, or mental status required general endotracheal anesthesia. Stereotactic instruments included the Nashold side-cutting biopsy needle (Integra, Plainsboro, NJ), $10 \mathrm{~mm}$ and $3 \mathrm{~mm}$ Sedan side-cutting biopsy needles (Elekta, Inc, Norcross, GA), and the Backlund aspiration needle system (Elekta, Inc, Norcross, GA). Biopsy technique was routinely planned to systematically sample tissue at both the edge of the lesion as well as the center of the lesion along the planned needle trajectory in a "geological core sample" manner at different depths, with multiple samples obtained at each depth by orienting the needle side opening in different radial directions (Fig. 1). All patients underwent immediate postprocedure CT imaging to assess the biopsy sight, were observed for $12 \mathrm{~h}$ in either an intensive care (2:1 nursing ratio) or a neuro-step-down critical care unit (3:1 nursing ratio), and most were routinely discharged home after one overnight hospital stay.

\section{Outcomes assessment}

Pathology diagnostic accuracy was assessed by success at arriving at a histological diagnosis (all lesions), as well as confirmation of the needle biopsy result after surgical resection of the lesion in those patients who underwent subsequent open craniotomy. Complications were assessed both clinically and by immediate post-biopsy CT imaging. All instances of bleeding through the needle(s) during the procedure were noted prospectively and characterized as: none, quickly clearing with 1-2 needle irrigations, or persisting beyond two needle irrigations. All new neurological deficits were noted as well as whether they were temporary, permanent, and/or required additional surgical intervention. Bleeding at the biopsy site on $\mathrm{CT}$ imaging was
Fig. 1 Left Schematic representation of the "Geologic Core" biopsy technique illustrating multiple biopsy depths (white lines) along a single needle trajectory. Right T2 weighted coronal MRI with biopsy plan. Multiple sample sites throughout the heterogeneous mass are indicated at 0,1 , and 2
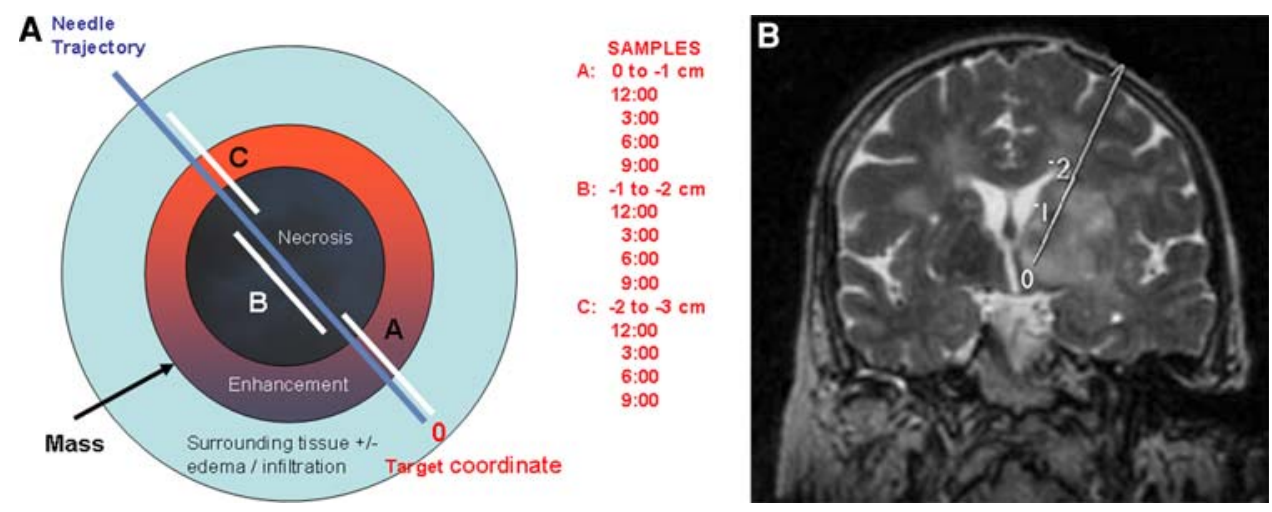
assessed and classified as $\leq 5 \mathrm{~mm}$ in diameter or $>5 \mathrm{~mm}$ in diameter, as well as whether or not it led to new neurological symptoms and/or required additional surgical intervention. The relationship of intra-operative findings of bleeding at the time of biopsy were correlated with the post-biopsy CT image findings by calculating the positive and negative predictive values for subsequent blood on CT scan using standard techniques [12].

Assessment of candidacy for frameless stereotactic alternative

All lesions were objectively measured on hard copy neuroimages with calipers, or on computer monitor screens with objective measurement tools, according to published techniques [13]. The anatomic location of each lesion as well as the distance relationship between the margin of the lesion and the arteries of the circle of Willis, the root of the Sylvian fissure, and the deep cerebral venous system (intracerebral veins and vein of galen), were noted, measured and recorded. In the portion of the study dedicated to determining the percentage of cases in this series that could definitely have been performed using a frameless stereotactic (FL) technique we assumed an average accuracy with of FL of $\pm 2-4 \mathrm{~mm}$. Lesions were excluded from FL technique consideration for safety concerns if they were (1) infratentorial or located in the pineal region, (2) located within $10 \mathrm{~mm}$ of an artery of the circle of Willis or the root of the Sylvian fissure, or (3) located within $10 \mathrm{~mm}$ of the deep cerebral venous system. Remaining lesions in the series were then divided into those $\geq 5 \mathrm{~mm}$ in diameter and those $\geq 10 \mathrm{~mm}$ in diameter for probability assessment for consistently hitting the target and obtaining the diagnosis assuming a central lesion target and average targeting error of $2 \mathrm{~mm}$ and $4 \mathrm{~mm}$, respectively.

\section{Results}

Median patient age was 55.5 years with a range from 3-81 years (Mean, 50 years; SD 21.7). There were 47 men and 44 women. Four patients had single lesions sampled on two different occasions separated by significant time. Four had two lesions, and one had eight lesions, addressed during the same procedure. One hundred lesions were approached for diagnostic biopsy, four were simple therapeutic cyst aspirations, and two were for installation of $\mathrm{P}^{32}$ for intracystic brachytherapy for craniopharyngioma. A summary of the technical aspects of the series is presented in Table 1 and the lesion locations and needle approached utilized are summarized in Table 2. For the diagnostic biopsy cases, the median number of separate samples submitted to pathology for each lesion was four (range, 2-16).
Table 1 Techniques utilized for 106 consecutive lesions

\begin{tabular}{lcl}
\hline Stereotactic system & & \\
Cosman-Roberts-Wells (CRW) & 68 & $64 \%$ \\
Leksell model G & 38 & $36 \%$ \\
Targeting imaging & & \\
$\quad$ Magnetic resonance imaging (MR) & 95 & $90 \%$ \\
Computed tomography (CT) & 11 & $10 \%$ \\
Anesthesia (95 procedures) & & \\
Local anesthesia with intravenous sedation & 68 & $71.6 \%$ \\
General endotracheal & 27 & $28.4 \%$ \\
$\quad$ Altered mental status & 14 & - \\
$\quad$ Pediatric (ages 3-13) & 8 & - \\
$\quad$ Posterior fossa sitting position & 3 & - \\
$\quad$ Language barrier & 1 & - \\
$\quad$ Patient choice & 1 & - \\
Entry technique & & \\
Twist drill craniostomy & 91 & $86 \%$ \\
Open burr hole & 15 & $14 \%$ \\
Posterior fossa sitting position & 3 & - \\
Brain stem lesion & 3 & - \\
Pineal region & 2 & - \\
Leksell temporal ring approach & 2 & - \\
Converted from twist drill & 1 & - \\
Other & 4 & - \\
\hline
\end{tabular}

Table 2 Lesion locations and approaches

\begin{tabular}{lrl}
\hline Lesion location & 33 & $(31.1 \%)$ \\
Single lobar & 25 & $(23.6 \%)$ \\
Multi-focal & 18 & $(17.0 \%)$ \\
Bi-hemispheric via corpus callosum & 7 & $(6.6 \%)$ \\
Multi-lobar (contiguous, uni-hemispheric) & 12 & $(11.3 \%)$ \\
Diencephalic & 8 & $(7.5 \%)$ \\
Brain stem & 3 & - \\
Midbrain & 2 & - \\
Midbrain and pons & 2 & - \\
Pons & 1 & - \\
Pons and medulla & 3 & $(2.8 \%)$ \\
Pineal region & & \\
Approaches & 51 & - \\
Perpendicular to convexity directly over lesion & 50 & - \\
Standard coronal suture entry & 3 & - \\
Posterior fossa—middle cerebellar peduncle & 2 & - \\
Forehead & & \\
\hline
\end{tabular}

Diagnostic yield and accuracy

Of the 100 consecutive diagnostic biopsies performed, a definite histological and/or microbiology diagnosis was obtained in 94 (94\% diagnostic yield). Of the six 
inconclusive cases, three demonstrated only necrosis on needle biopsy and we went on to confirm Glioblastoma multiforme (GBM) at subsequent surgery (two repeat needle biopsies, one open craniotomy). Two were suggestive but not definitive for bacterial abscess and responded to empiric antibiotic treatment. One was shown to be tumescent multiple sclerosis (MS) on subsequent open biopsy. The diagnoses obtained are outlined in Table 3.

Eleven of the 94 diagnostic biopsy patients where we obtained a definite histological or microbiology diagnosis on needle biopsy subsequently underwent open craniotomy within two months of the original biopsy. Review of these pathology results against those obtained with the stereotactic needle biopsy revealed that the larger sampling size of the open craniotomy confirmed the original stereotactic needle biopsy result in ten of eleven cases $(90.9 \%$ accuracy). The only outlier was a single patient thought to have a low grade Oligodendroglioma on stereotactic needle biopsy who subsequently was confirmed to have a higher grade tumor (anaplastic oligodendroglioma).

\section{Corpus callosum and multifocal lesions}

Of the eighteen lesions involving the corpus callosum, 13 (72.2\%) turned out to be GBM, but two were found to be anaplastic astrocytoma, and one each were found to be

Table 3 Diagnoses in 100 consecutive needle biopsies

\begin{tabular}{lr}
\hline WHO Grade IV Astrocytoma & 26 \\
WHO Grade III Astrocytoma & 5 \\
WHO Grade II Astrocytoma $^{\mathrm{a}}$ & 8 \\
WHO Grade I Astrocytoma (Pilocytic) & 2 \\
Oligodendroglioma & 9 \\
Anaplastic oligodendroglioma & 6 \\
Anaplastic oligo-astrocytoma & 1 \\
Central nervous system lymphoma & 4 \\
Metastatic brain tumor & 7 \\
Hamartoma & 2 \\
Immature teratoma & 1 \\
Germinoma & 1 \\
Meningioma & 1 \\
Radiation necrosis & 3 \\
Tumescent multiple sclerosis & 3 \\
Hematoma cavity & 2 \\
Arteriovenous malformation & 1 \\
Bacterial abscess & 10 \\
Progresssive multifocal leukoencephalopathy & 1 \\
Viral encephalitis & 1 \\
Inconclusive & 6 \\
\hline WHO Wort &
\end{tabular}

WHO World Health Organization

a Three of the eight grade II tumors were gemistocytic astrocytomas anaplastic oligodendroglioma, primary central nervous system lymphoma (PCNSL) and tumescent MS, respectively. Altogether, $27.8 \%$ of these cases turned out not to have GBM. Of the 25 multifocal lesions in our series, only one turned out to have abscesses and only three had metastatic brain tumors (two of the three with no known primary). While radiation necrosis was the diagnosis for 2 $(8 \%)$, a malignant primary brain tumor was the cause of the multifocal lesions in 17 (68\%) (11 GBM, three PCNSL, two anaplastic ologodendroglioma, and one anaplastic astrocytoma).

Complications and predictive value of intra-operative findings

Intraoperative bleeding from the twist drill craniostomy puncture only required conversion to open burr hole in one instance $(1 / 92=1.1 \%)$. In this instance, a bleeding surface vessel branch was identified and cauterized. Post-biopsy CT images did not reveal a single instance of entry site hemorrhage in the other 91 instances. The incidence of identifying any intraparenchymal blood at the biopsy site on post-biopsy CT images is outlined in Table 4 and examples are presented in Fig. 2.

Some amount of blood was identified in the needle or the specimen during the procedure in 23 cases. Continued bleeding that required persistence of the needle at the biopsy site and irrigation and clearing of the needle until bleeding spontaneously ceased was present in 19 instances (19\%). There were no cases where the bleeding through the needle did not eventually stop with continuing irrigation. For the subsequent finding of any blood at all at the biopsy site on post-biopsy CT, the presence of persistent bleeding beyond two needle irrigations intraoperatively has a positive predictive value of $63.2 \%$ and a negative predictive value of $86.4 \%$. For the subsequent finding of $>5 \mathrm{~mm}$ of blood at the biopsy site on post-biopsy CT, the presence of this finding intraoperatively has a positive predictive value of only $21.1 \%$, but a very powerful negative predictive value of $98.8 \%$. Thus if there is no persistent bleeding beyond two needle irrigations intraoperatively, there is only a $1.2 \%$ chance of identifying blood $>5 \mathrm{~mm}$ diameter on post-biopsy neuroimaging.

Table 4 Post-needle biopsy CT scan findings and significance in 100 consecutive cases

\begin{tabular}{lrl}
\hline No hemorrhage noted on CT & 83 & $(83 \%)$ \\
Small $\leq 5 \mathrm{~mm}$ area of hemorrhage & 18 & $(18 \%)$ \\
$>5 \mathrm{~mm}$ area of hemorrhage & 5 & $(5 \%)$ \\
New temporary neurological deficit & 3 & $(3 \%)$ \\
New permanent neurological deficit & 1 & $(1 \%)$ \\
Additional surgery due to hemorrhage & 0 & $(0 \%)$ \\
\hline
\end{tabular}


Fig. 2 Examples of post-biopsy hemorrhage (arrows). Left A small $(<5 \mathrm{~mm})$, clinically silent hemorrhage adjacent to postbiopsy air within the left frontal subcortical white matter. Right A larger hemorrhage within the deep white matter in a patient with temporary worsening of her left hemiparesis following biopsy
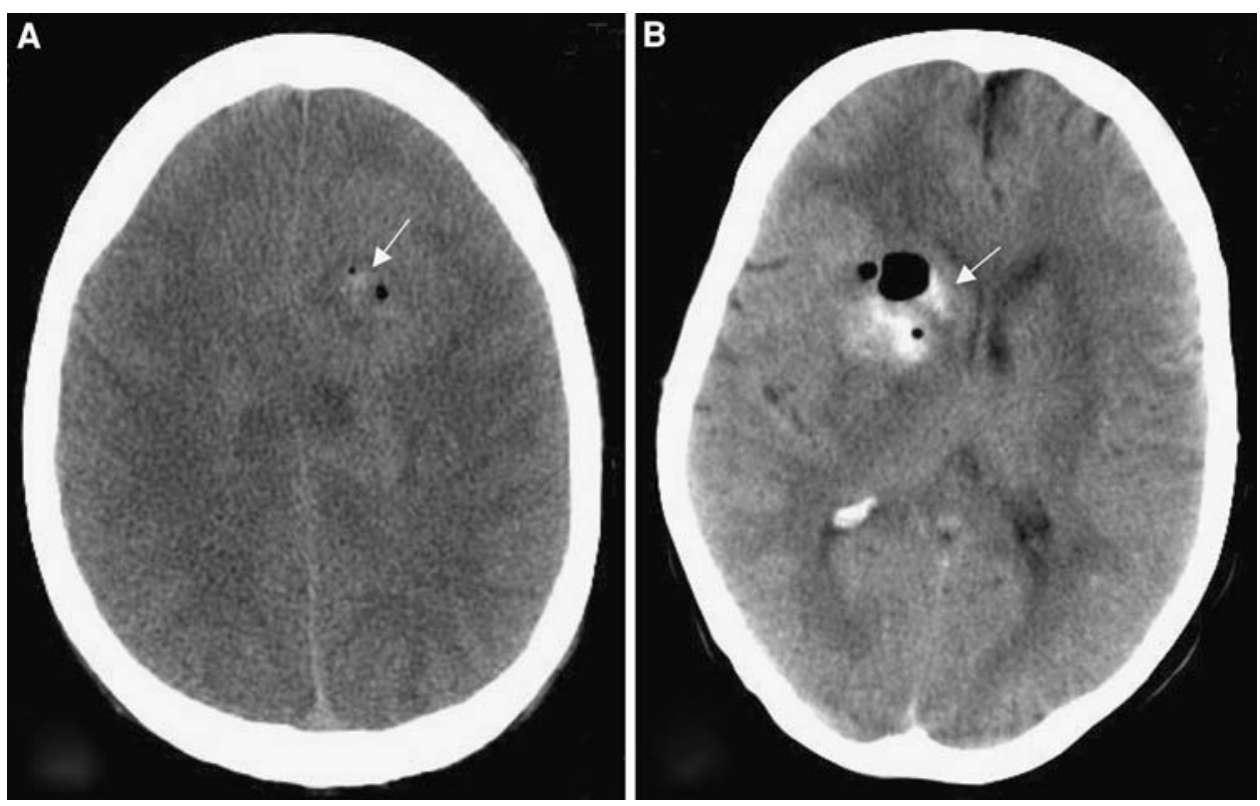

Clinically, only three patients developed new temporary neurological symptoms or signs and only one developed permanent new neurological symptoms or signs (Table 4). The temporary clinical complications included headache in one (pineal region tumor), new partial hemiparesis in one and new temporary double vision in a patient with a midbrain lesion. The permanent complication was permanent diplopia and subtle worsening of pre-existing hemiparesis in a patient with a midbrain malignant glioma. No patient required operation for cerebrospinal fluid diversion or for hematoma evacuation as a result of stereotactic biopsy. Clinical complication risk was higher for patients with brain stem lesions (12.5\% temporary and another $12.5 \%$ mild permanent) and pineal region lesions $(33.3 \%$ temporary, $0 \%$ permanent) versus patients with non-brain stem or pineal region lesions (1.1\% temporary and $0 \%$ new permanent).

Proportion of cases that would have been candidates for frameless stereotactic technique

Of our 100 consecutive diagnostic biopsy cases, eleven arose in the midbrain, pons or pineal region, and seven were located within $10 \mathrm{~mm}$ of an artery of the circle of Willis or the root of the Sylvian fissure, or within $10 \mathrm{~mm}$ of the deep cerebral venous system (see Figs. 3, 4 and 5 for examples). In a paradigm where a lesion had to satisfy these anatomic location constraints as well as be at least
Fig. 3 a Axial targeting MR (T1-weighted with gadolinium contrast) in an adult with a nonenhancing anaplastic astrocytoma of the whole pons. Note that the CRW frame is rotated to provide direct access to a left middle cerebellar peduncle needle trajectory without interfering with the left posterior frame post. b Postbiopsy non-contrast CT scan demonstrating the open burr whole entry point (arrow) as well as the $2 \mathrm{~cm}$ core biopsy site (between asterisks)
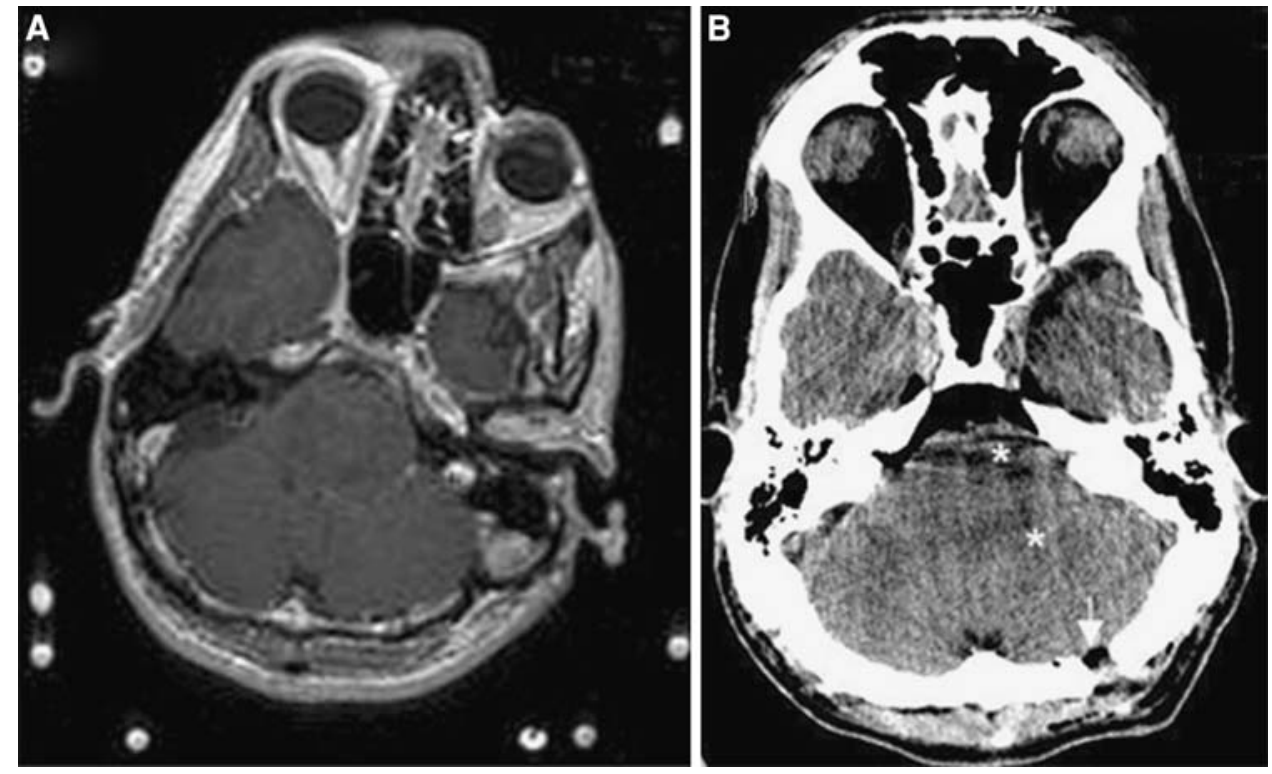

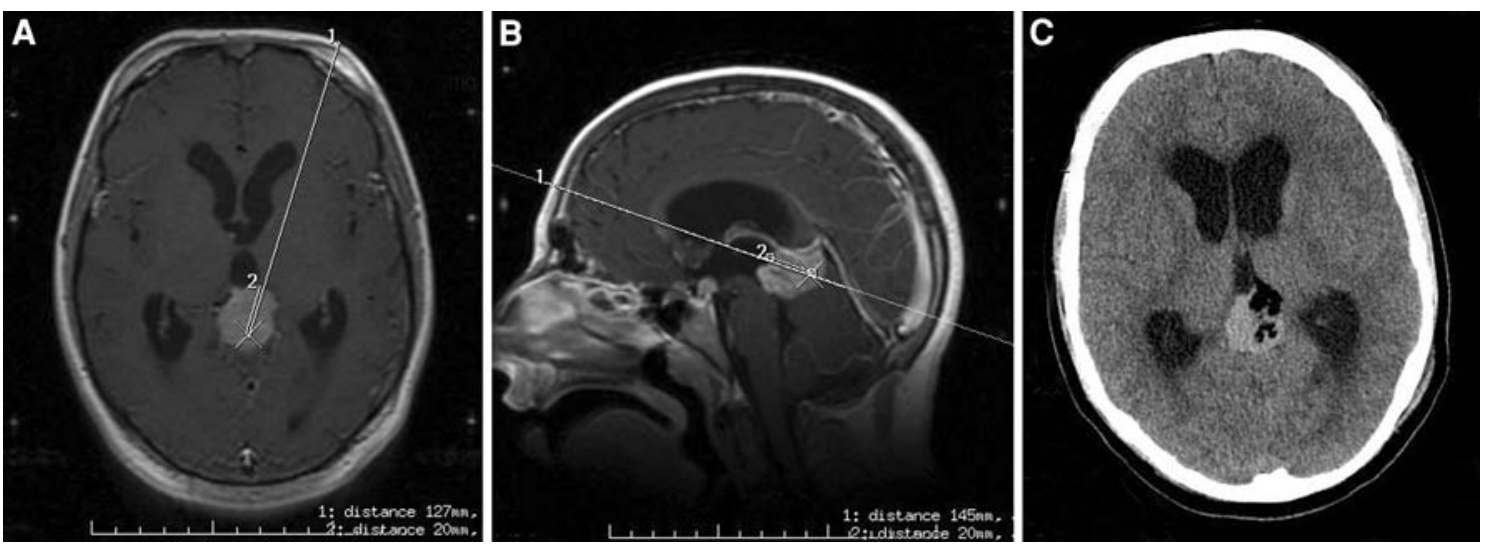

Fig. 4 a Axial targeting MR (T1-weighted with gadolinium contrast) in an adult with an enhancing pineal region mass showing the left forehead twist drill craniostomy needle trajectory and the planned $2 \mathrm{~cm}$ core biopsy site using a Leksell $\mathrm{G}$ frame. b Sagittal targeting MR (T1-weighted with gadolinium contrast) again demonstrating the forehead twist drill craniostomy needle trajectory and the planned
$2 \mathrm{~cm}$ core biopsy site, but also demonstrating how close the target is to the internal cerebral vein, the vain of Galen and the straight sinus. c Post-biopsy non-contrast CT scan with air defects at the biopsy site. The lesion is intrinsically mildly hyperdense throughout and no bleeding resulted from the biopsy
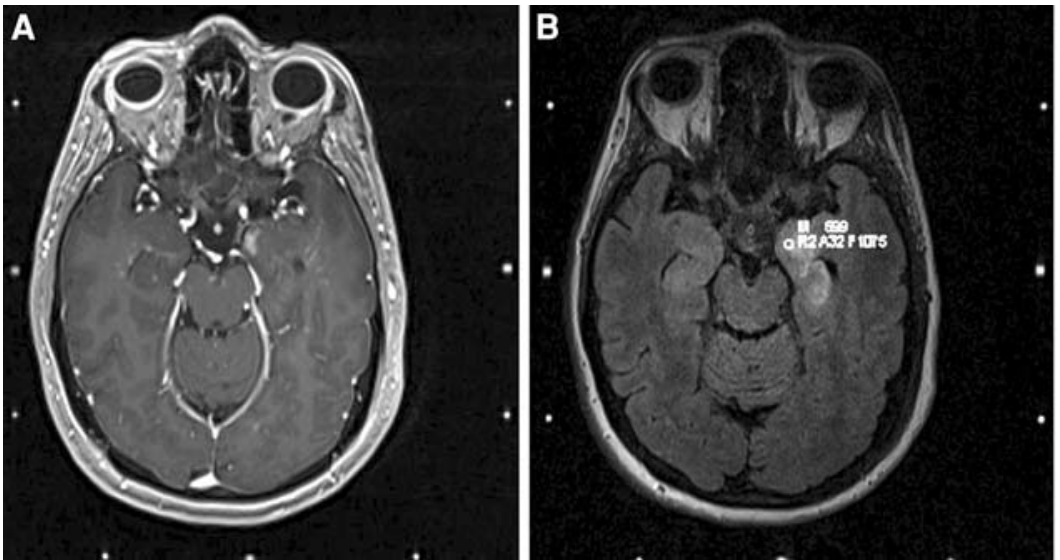

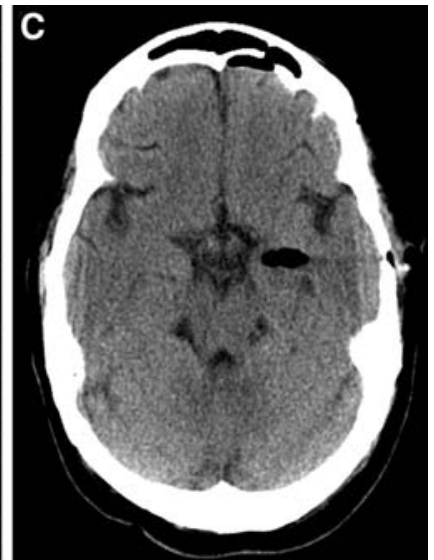

targeting MR demonstrating a larger area of lesion signal change as well as the deep target point. c Post-biopsy non-contrast CT scan with air defect indicating the $2 \mathrm{~cm}$ core biopsy tract without any postbiopsy bleeding

\section{Discussion}

$5 \mathrm{~mm}$ in size for eligibility, $82 \%$ of the 100 lesions in our series would have been candidates for FL needle biopsy as an alternative to the FB technique. No lesion would be excluded for size concerns. In a paradigm where a lesion had to satisfy these anatomic location constraints as well as be at least $10 \mathrm{~mm}$ in size for targeting likelihood, $80 \%$ of the 100 lesions in our series would have been candidates for FL needle biopsy as an alternative to the frame-based technique. Four lesions would have been excluded for size and targeting accuracy concerns (two of which were already excluded for anatomic location concerns. All together, based on these assumptions $80-82 \%$ of the lesions in our series would have been candidates for FL needle biopsy as an alternative to the FB technique.
The reliability of frame-based stereotactic biopsy

Overall, our data support the notion that stereotactic biopsy is an effective means of establishing tissue diagnosis for intracranial lesions. Our diagnostic yield of $94 \%$ compared favorably with recent and historical large series reporting ranges of $90-98 \%$ (Table 5). Even for the 6\% of our cases with nondiagnostic results, tissue pathology was still suggestive enough to effectively guide treatment in all but one instance. In our series, lesion size and/or location did not correlate with diagnostic yield or accuracy. 
Table 5 Summary of large stereotactic series

\begin{tabular}{|c|c|c|c|c|c|c|c|c|}
\hline Author & Center & Year & System & $\mathrm{N}$ & DY $(\%)$ & $\begin{array}{l}\text { DA } \\
(\%)\end{array}$ & $\begin{array}{l}\text { Morbidity } \\
(\%)\end{array}$ & $\begin{array}{l}\text { Mortality } \\
(\%)\end{array}$ \\
\hline Ostertag [1] & $\begin{array}{c}\text { University of } \\
\text { Friedburg }\end{array}$ & 1980 & $\begin{array}{l}\text { Reichart Mundinger } \\
\text { CT indexed to } \\
\text { intra-operative } \\
\text { ventriculogram }\end{array}$ & 302 & $>90$ & NR & 2.3 & 3 \\
\hline Edner [2] & Karolinska & 1981 & Leksell & 345 & $\begin{array}{c}91 \text { if ct } \\
\text { used }\end{array}$ & $?$ & $<1$ & 2.3 \\
\hline Lobato [3] & Madrid & 1982 & Leksell & 100 & 97 & NR & 7 & 0 \\
\hline Apuzzo [5] & USC & 1983 & BRW & 83 & 94 & NR & 4 & 0 \\
\hline Lunsford[6] & Pittsburgh & 1984 & Leksell & 102 & 96.5 & NR & 3 & 0 \\
\hline Mundinger [7] & Friedburg & 1985 & Mundinger & 815 & & & 3 & 0.6 \\
\hline Apuzzo [4] & USC & 1987 & BRW & 500 & 95.6 & $?$ & 1 & 0.2 \\
\hline Bernstein [20] & Toronto & 1986-1994 & BRW & 300 & NR & NR & 6.3 & 1.7 \\
\hline Grunert [15] & JGU & 1994 & $\mathrm{BRW/CRW}$ & 200 & 92 & 91 & 3 & 1 \\
\hline Regis [19] & $\begin{array}{l}\text { Centre Hospitalier } \\
\text { Universitaire } \\
\text { la Timone, } \\
\text { Marseille, France }\end{array}$ & 1996 & $\begin{array}{l}\text { 15centers: Talairach } 8 \\
\text { Leksell } 2 \text { both } \\
2 \text { other } 3\end{array}$ & 370 & 94 & 97.7 & $\begin{array}{l}0.8 \text { sig. } \\
7 \text { transient }\end{array}$ & 1.3 \\
\hline Ulm,Friedman [17], & Gainesville & 2001 & & 200 & 98.5 & & 2 & 0 \\
\hline Field, Kondziolka[18] & Pittsburgh & 2001 & Leksell & 500 & NR & NR & $\begin{array}{l}1.28 \% \text { radiographic } \\
\text { hemorrhage }\end{array}$ & 0.2 \\
\hline Smith,McDermot [10] & UCSF & 2006 & CRW or BRW & $213^{\mathrm{a}}$ & 90 & NR & 2 & 0 \\
\hline Woodworth et al. [11] & Johns-Hopkins & 2006 & Leksell or CRW & $160^{\mathrm{a}}$ & 91 & NR & 13 & 1 \\
\hline Linskey (present) & U. Arkansas & 2007 & CRW or Leksell & 106 & 96 & 91 & 4 & 0 \\
\hline
\end{tabular}

$N R$ Not reported, BRW Brown-Roberts-Wells, CRW Cosman-Roberts-Wells

${ }^{\text {a }}$ Frame-based arm only

Diagnostic accuracy is an equally important consideration in evaluating the utility of stereotactic biopsy, though it is reported far less frequently in the literature than diagnostic yield. At least one study has suggested that biopsy specimens cannot provide a sufficient accuracy of diagnosis to reliably guide treatment of brain neoplasms, citing discrepancies as high as $38-49 \%$ when biopsies specimens were compared with final pathological diagnosis obtained at open surgical resection [14]. Those figures, however, were based upon biopsies performed by multiple outside facilities and few details regarding the specific techniques used are provided. Large single center series with higher individual procedure volumes are likely more representative of the true potential of stereotactic biopsy, and a thorough and systematic biopsy technique is necessary for optimal results. Our accuracy rate of $90.9 \%$ is in keeping with that of Grunert et al. [15], who reported a diagnostic accuracy of $91 \%$ in 47 patients who underwent a subsequent open resection. Woodworth et al. [16] reported an accuracy rate of $76 \%$, though results correctly guided treatment in $91 \%$ of their series of 21 patients who underwent open biopsy. Our single inaccuracy was a WHO II oligodendroglioma which was subsequently found to be WHO III at the time of resection. While we did not detect this tendency, a few reports have suggested that mixed gliomas with a significant oligodendroglial component may be more commonly mis-graded on stereotactic biopsy than other glioma histologies $[14,16]$.

The specific method of tissue biopsy likely plays a key role in determining diagnostic accuracy and yield. Past series appear to be relatively evenly divided between the use of biopsy forceps $[1,4]$ or a side-cutting biopsy needle $[17,18]$ while many report using both $[15,19,20]$, and still other surgeons still use needle aspiration techniques or the Backlund spiral devise (Elekta, Inc, Norcross, GA). More recent series tend to favor a side-cutting needle exclusively $[10,11,18]$, which has the advantage of preserving a core of intact cross-sectional tissue architecture which facilitates histological interpretation. The present series used a relatively aggressive biopsy technique which we feel minimizes sampling error and increases the likelihood of an accurate diagnosis. When practical, a target point beyond the edge of the lesion was selected. Multiple sections were then taken with the side-cutting needle at serial depths along the track. In this manner a "geologic core" could be obtained with a single needle trajectory, providing samples of normal brain, lesion edge, and central contents. The utility of this approach is reflected in the accurate 
grading of all but one of the gliomas in our series, and in the low number (three) of necrosis-only results in our GBM biopsies.

\section{Role of stereotactic biopsy in clinical decision making}

One particularly important role for stereotactic biopsy is confirming tissue diagnosis for patients with multiple brain lesions, specifically in the setting of a negative systemic metastatic survey. Of our 25 multifocal lesions, a significant majority (68\%) were primary CNS neoplasms, reinforcing the importance of tissue diagnosis when no obvious metastatic source can be found. Not all patients with multiple CNS brain lesions can be assumed to have metastatic disease. Even higher instances of multifocal primary CNS disease have been reported elsewhere. Yamada et al. [21] found zero metastases out of $25 \mathrm{mul}-$ tifocal brain lesions that were referred for stereotactic biopsy. All three patients with multifocal lesions described in Lunsford et al. [6] were found to have gliomas. In all, Lunsford also found that nearly $10 \%$ of pre-procedure diagnoses classified as "secure" were overturned after biopsy. Another nine out of 44 patients with a "strongly suspected" pre-op diagnosis were found to have a pathology that was not considered in the pre-biopsy differential. A retrospective review by Arbit and Galicich [22] similarly found that results of stereotactic biopsy dictated different treatment than radiographic diagnosis in 19\% of cases.

Our data also indicate that stereotactic biopsy is an important tool for establishing the diagnosis of corpus callosum lesions. Conventional teaching has often been that patients with lesions crossing the corpus callosum do not have resectable lesions and can be assumed to have GBM, and thus can be empirically treated. While most of the 18 patients in our series with a callosal lesion did turn out to have GBM, 27.8\% had lesions which mandated different management, including oligodendroglioma, PCNSL, and tumescent MS. This finding becomes particularly important given that some authors have questioned the utility of biopsy in the management of gliomas [14]. In that study, only three out of 81 lesions were located in the corpus callosum. The present data indicate that a diagnosis of glioma based solely upon characteristic imaging is premature without a tissue diagnosis.

\section{Safety considerations in stereotactic biopsy}

It is of interest that the morbidity rates reported in recent stereotactic series differ little from those performed nearly three decades ago (Table 5). Mortality rates, in contrast, have tended to decline slightly over the same period, possibly through technological and infrastructure improvements which allow for faster recognition and correction of post- procedural emergencies. Our morbidity rate of $4 \%$ (temporary or permanent neurologic deficit) corroborates the results of these other large series and also demonstrates that an aggressive sampling technique can be employed without compromising patient safety. This is consistent with the study of Mcgirt et al. [23], which found that increasing the number of biopsy samples did not independently impact morbidity if the samples were collected along a single needle trajectory.

Brainstem and pineal locations accounted for three of four complications, and this is consistent with the findings of previous authors who correlated morbidity with pineal [18] and deep-seated lesions [23]. In general, relatively small numbers of pineal locations in this and other series make generalization to an accurate risk profile for these lesions difficult. A contrary view was offered by Regis et al. [19], who reported the results of a multicenter series of 370 pineal region stereotactic biopsies and found that complication rates were no higher than in other locations if only permanent deficits were considered. That study did note an increased likelihood of complication associated with "hard" tumors (pineocytomas, teratomas, and astrocytomas) and recommends proceeding with a microsurgical approach in the event that tissue is not easily obtained with the first pass of the biopsy needle.

In seeking possible predictors of post-procedure complication, we have specifically identified the finding of blood within the biopsy needle that persists beyond two needle irrigations. Shastri-Hurst et al. [24] have previously noted the finding of blood intra-operatively in 7/203 cases as having a positive predictive value of $57 \%$ for postoperative deterioration but a sensitivity of only $30 \%$. In our series, $19 \%$ of patients met our particular criteria for persistent intra-operative bleeding. We found the absence of this finding in the remaining $81 \%$ to have a very high $(98.6 \%)$ negative predicative value for a significant ( $>5 \mathrm{~mm}$ ) hemorrhage being identified on the post-operative CT. Our analysis of the Shastri-Hurst et al. data reveals a similar negative predicative value $(95.4 \%)$ for post-procedural deterioration. With such high negative predicative values, routine post-biopsy neuroimaging in the absence of bleeding through the needle persisting beyond two irrigations or development of a new neurological deficit can probably be safely eliminated from stereotactic needle biopsy patient care protocols.

While some authors have suggested that patients with normal postoperative scans do not require further assessment [25], Field et al. [18] found a small but non-zero incidence $(0.4 \%)$ of delayed neurologic deterioration after uncomplicated brain biopsy with negative post-operative imaging. This leaves the question of an appropriate level of nursing care for these patients still open to debate. It is our opinion that a step-down level facility or well trained 
neurosurgical floor capable of providing neurologic assessments every $2 \mathrm{~h}$ is sufficient for the initial 12 postoperative hours in uncomplicated cases. In other publications, poor glycemic control in diabetics [23] and platelet counts $<150,000$ [18] have also been identified as statistically significant independent risk factors for poor outcome after stereotactic biopsy and should be considered in postbiopsy management decisions. .

Frameless stereotaxy: a hypothetical cohort

Given the increasing popularity of frameless neuro-navigation systems in stereotactic biopsy, we sought to establish criteria through which we could determine the suitability of frameless stereotaxis (FL) for patients in our series. We began with the assumption that frame-based stereotaxis (FB) represents the gold standard for targeting accuracy and examined the literature for assessments of FB and FL systems.

In reviewing the spatial accuracy of various FB and FL systems, it is important to understand the practical significance of the different measures of accuracy commonly cited. Intraoperative computer workstations provide an estimate of root mean square error (RMS) following coregistration of skin fiducials or the stereotactic headframe. However this value should not be considered indicative of true accuracy. Rather, RMS represents the degree of internal consistency between data points-in this case the computed coordinates within the virtual space of the computer workstation. RMS gives no information regarding the correspondence of those coordinates to the actual location of objects in physical space. This concept was elegantly demonstrated by Mascott et al. who found no statistically significant correlation of RMS values with the accuracy of marker placement in a large in vivo study [26]. Studies using phantom models typically measure the mean error of localization, which represents the average magnitude of the distance between the probe and its intended target. This should not be confused with the mean errors reported for individual axes in some in vivo [27] and phantom [28] studies which utilized planar imaging to measure targeting accuracy. In such instances mean errors refers to the average error within a single anatomic plane. A Euclidean error is then calculated as the square root of the sum of the squares of the mean errors in each dimension. Euclidean error is therefore generally larger than mean error, and more representative of the actual distance from a target one could reliably expect to achieve.

FB has traditionally been touted as being capable of submillimeter accuracy, though recent studies suggest that this may not be an entirely realistic expectation. Hall et al. [29] found a euclidean error of $1.53 \mathrm{~mm}$ using a Leksell frame and MRI imaging in a phantom model. In a large phantom model study, Maciunas et al. [30] found that while the mean mechanical errors of the CRW and BRW frames were less than $1 \mathrm{~mm}$, at a $99.9 \%$ confidence interval they can only be expected to achieve a mechanical accuracy of $2 \mathrm{~mm}$ or less. When factors such as imaging, point selection, and vector calculations were all considered, the "application error" at the $99.9 \%$ confidence interval increased to $3.1-5.0 \mathrm{~mm}$ for four different frame systems. It is perhaps not practical, though, to make estimates of accuracy based upon an extreme limit of error that is likely to occur once in 1,000 cases. For this same series, the mean error of localization, or average distance between the probe and its intended target, was between 1.2 and $1.9 \mathrm{~mm}$ for the various systems tested. We feel that this is a more realistic estimate of the sort of accuracy one can expect in a given procedure. This corresponds well with in vivo assessments of FB for deep brain stimulator targeting accuracy, which found average stereotactic errors of 1.4$2 \mathrm{~mm}[31]$.

There are several factors which likely contribute to the inherently higher accuracy of FB. These systems have their frame of reference rigidly fixed to the skull and established as soon as the frame is applied. Fiducial markers applied to skin are inherently more mobile and must be re-referenced to the navigation system once the patient is positioned, introducing two potential sources of error. Errors of imprecise trackable probe positioning as well as computer cursor positioning can be reduced, but not eliminated. The location of the fiducials has a significant impact on the zone of maximal correlation which increases accuracy and thus accuracy can vary considerably through various locations in a given registered target volume. The use of anatomic surface landmark registration in lieu of fiducial placement has consistently demonstrated lower accuracy rates [32], particularly for posterior lesions where reliable anatomic landmarks are fewer.

In vivo assessments of FL systems have tended to yield larger error measurements than FB. Dorward et al. [27] found a euclidean error of $4.8 \mathrm{~mm}$ in the in vivo arm of their study. Mascott et al. [26] report mean localization errors between 3.3 and $5.4 \mathrm{~mm}$. One relatively recent comparative phantom study actually found a smaller euclidean error in a frameless system when a specific planning and targeting protocol was used (probe's eye) [28]. It must be noted, as those authors themselves attest, that skull phantom models tend to overestimate the accuracy of FL systems, since such models simulate placing the fiducials directly upon the skull rather than the overlying skin, eliminating one major source of error in skin fiducial based systems. Indeed, in the Mascott study, mean localization error fell to $1.4-1.9 \mathrm{~mm}$ when skull impanted fiducials were used. Continued investigation in this realm is important as advances in computer image processing and algorithms enable 
neuronavigational systems to gain ground on the relatively established frame-based technologies. However, the above investigations indicate that currently, while it may be unrealistic to assume submillimetric accuracy for FB systems, it is probably reasonable to expect reliable targeting within 1-1.5 mm. Applying similar standards to FL systems using skin fiducials, that expected error rises to 3-4 $\mathrm{mm}$ and occasionally even greater depending on registration technique.

With this in mind, we devised our criteria of potential candidacy for FL biopsy. We found that fully $80 \%$ of our patients were candidates for frameless approach. Depending on which estimates of accuracy are used, FL systems can be expected to reliably target lesions of $>5-10 \mathrm{~mm}$. Grunert et al. [33] came to a more conservative conclusion and suggested that lesions less than $15 \mathrm{~mm}$ should be reserved for FB approach. From a practical standpoint, requiring a lesion diameter of at least $10 \mathrm{~mm}$ rather than $5 \mathrm{~mm}$ excluded only two patients from the FL eligible group.

While based on accuracy considerations alone, $>80 \%$ of biopsies could be accomplished through FL techniques, there are less easily quantifiable variables which ultimately influence technique selection. The risks of general anesthesia need to be considered, and the FB technique has traditionally been accomplished with local anesthesia and sedation. However in at least two comparative series, the surgeons induced general anesthesia in all patients $[8,11]$. This clearly influenced the outcome measure of total time and cost, which both studies found to favor FL. A comparative series conducted by Smith et al. [10] which used local anesthetic for the FB arm found a substantial reduction in operating room time and cost with FB. While we utilized general anesthesia in $28.4 \%$ of cases, the remainder were done under local anesthesia. Frame-based stereotaxis has also typically been considered less invasive as it can usually be performed through a tiny stab incision and twistdrill craniostomy. While the FL technique could theoretically be performed through a twist drill craniostomy, rather than a burr hole, most FL technique series report the use of burr holes. Regardless, the three-point skull screw fixation, locking-ball-socket, device usually used for FL technique requires an actual incision to expose enough skull surface to seat the device and cannot be inserted through a simple stab wound. While $14 \%$ of our cases did require an incision and an open burr hole, this more invasive approach was safely avoided in the remaining $86 \%$. Given the similarity of more objective measures such as diagnostic yield and complication rate between the two techniques $[10,11]$, such discrepancies serve to highlight the important role individual surgeon training, preference and experience have in determining technique selection. For diagnosis of small deep-seated lesions, familiarity with frame-based techniques remains an important tool.

\section{Conclusions}

Frame-based stereotaxis through a systematic "core-sampling" technique is a safe, reliable and valuable tool for obtaining tissue diagnosis and directing future therapy. Stereotactic biopsy is particularly valuable in the evaluation of patients with multiple brain lesions in the absence of a known metastatic source, and for lesions involving the corpus callosum. The absence of persistent blood in the biopsy needle at the time of surgery has a high negative predicative value for the presence of hemorrhage on postoperative CT imaging. Post-operative CT scanning can probably be safely reserved for those patients who demonstrate persistent bleeding through the needle after several irrigation clearing attempts or develop new neurological deficits. Frame-based stereotaxis remains the gold-standard for targeting accuracy, though in practice $>80 \%$ of lesions can be safely and effectively approached through frameless biopsy with neuro-navigation systems. Frame-based technique is indicated for approaching brainstem, pineal, and deep peri-vascular lesions. For larger and more superficial lesions, less easily quantifiable considerations such as surgeon preference and institution specific protocols and infrastructure are more immediately important determinants. Given the current state of the art, Frame-based stereotaxis remains an important technique, though one of fading prominence in the evolving frameless era.

Acknowledgments The clinical work reported in this study was performed at the University of Arkansas for Medical Sciences at the University Hospital, Children's Hospital of Arkansas, and the McClellan VA Medical Center in Little Rock, AR. Steve Davis, RN participated in the prospective database maintenance and periodic update as well as neuroimaging collation. Christopher Owen is supported by a research fellowship from the Department of Neurological Surgery at the University of California, Irvine.

Open Access This article is distributed under the terms of the Creative Commons Attribution Noncommercial License which permits any noncommercial use, distribution, and reproduction in any medium, provided the original author(s) and source are credited.

\section{References}

1. Ostertag CB, Mennel HD, Kiessling M (1980) Stereotactic biopsy of brain tumors. Surg Neurol 14:275-283

2. Edner G (1981) Stereotactic biopsy of intracranial space occupying lesions. Acta Neurochir (Wien) 57:213-234. doi:10.1007/ BF01664839

3. Lobato RD, Rivas JJ, Cabello A, Roger R (1982) Stereotactic biopsy of brain lesions visualized with computed tomography. Appl Neurophysiol 45:426-430. doi:10.1159/000101637

4. Apuzzo ML, Chandrasoma PT, Cohen D, Zee CS, Zelman V (1987) Computed imaging stereotaxy: experience and perspective related to 500 procedures applied to brain masses. Neurosurgery 20:930-937. doi:10.1097/00006123-198706000-00019 
5. Apuzzo ML, Sabshin JK (1983) Computed tomographic guidance stereotaxis in the management of intracranial mass lesions. Neurosurgery 12:277-285. doi:10.1097/00006123-19830300000005

6. Lunsford LD, Martinez AJ (1984) Stereotactic exploration of the brain in the era of computed tomography. Surg Neurol 22:222230. doi:10.1016/0090-3019(84)90003-X

7. Mundinger F (1985) CT stereotactic biopsy for optimizing the therapy of intracranial processes. Acta Neurochir Suppl (Wien) 35:70-74

8. Dorward NL, Paleologos TS, Alberti O, Thomas DG (2002) The advantages of frameless stereotactic biopsy over frame-based biopsy. Br J Neurosurg 16:110-118. doi:10.1080/0268869022 0131705

9. Paleologos TS, Dorward NL, Wadley JP, Thomas DG (2001) Clinical validation of true frameless stereotactic biopsy: analysis of the first 125 consecutive cases. Neurosurgery 49:830-835 discussion 835-837

10. Smith JS, Quinones-Hinojosa A, Barbaro NM, McDermott MW (2005) Frame-based stereotactic biopsy remains an important diagnostic tool with distinct advantages over frameless stereotactic biopsy. J Neurooncol 73:173-179. doi:10.1007/s11060004-4208-3

11. Woodworth GF, McGirt MJ, Samdani A, Garonzik I, Olivi A, Weingart JD (2006) Frameless image-guided stereotactic brain biopsy procedure: diagnostic yield, surgical morbidity, and comparison with the frame-based technique. J Neurosurg 104:233-237. doi:10.3171/jns.2006.104.2.233

12. Sackett DL (1997) Evidence based medicine: how to practice and teach EBM. Churchill Livingstone, New York

13. Linskey ME, Lunsford LD, Flickinger JC (1991) Neuroimaging of acoustic nerve sheath tumors after stereotaxic radiosurgery. AJNR Am J Neuroradiol 12:1165-1175

14. Jackson RJ, Fuller GN, Abi-Said D, Lang FF, Gokaslan ZL, Shi WM, Wildrick DM, Sawaya R (2001) Limitations of stereotactic biopsy in the initial management of gliomas. Neuro-oncol 3:193200. doi:10.1215/15228517-3-3-193

15. Grunert P, Ungersbock K, Bohl J, Kitz K, Hopf N (1994) Results of 200 intracranial stereotactic biopsies. Neurosurg Rev 17:5966. doi:10.1007/BF00309989

16. Woodworth G, McGirt MJ, Samdani A, Garonzik I, Olivi A, Weingart JD (2005) Accuracy of frameless and frame-based image-guided stereotactic brain biopsy in the diagnosis of glioma: comparison of biopsy and open resection specimen. Neurol Res 27:358-362. doi:10.1179/016164105X40057

17. Ulm AJ, Bova FJ, Friedman WA (2001) Stereotactic biopsy aided by a computer graphics workstation: experience with 200 consecutive cases. Surg Neurol 56:366-371 discussion 371-362

18. Field M, Witham TF, Flickinger JC, Kondziolka D, Lunsford LD (2001) Comprehensive assessment of hemorrhage risks and outcomes after stereotactic brain biopsy. J Neurosurg 94:545-551

19. Regis J, Bouillot P, Rouby-Volot F, Figarella-Branger D, Dufour H, Peragut JC (1996) Pineal region tumors and the role of stereotactic biopsy: review of the mortality, morbidity, and diagnostic rates in 370 cases. Neurosurgery 39:907-912 discussion 912-904
20. Bernstein M, Parrent AG (1994) Complications of CT-guided stereotactic biopsy of intra-axial brain lesions. J Neurosurg $81: 165-168$

21. Yamada K, Goto S, Kochi M, Ushio Y (2004) Stereotactic biopsy for multifocal, diffuse, and deep-seated brain tumors using Leksell's system. J Clin Neurosci 11:263-267. doi:10.1016/ j.jocn.2003.03.004

22. Arbit E, Galicich JH (1994) Importance of image-guided stereotactic biopsy to confirm diagnosis in an oncological setting. Ann Surg Oncol 1:368-372. doi:10.1007/BF02303807

23. McGirt MJ, Woodworth GF, Coon AL, Frazier JM, Amundson E, Garonzik I, Olivi A, Weingart JD (2005) Independent predictors of morbidity after image-guided stereotactic brain biopsy: a risk assessment of 270 cases. J Neurosurg 102:897-901

24. Shastri-Hurst N, Tsegaye M, Robson DK, Lowe JS, Macarthur DC (2006) Stereotactic brain biopsy: an audit of sampling reliability in a clinical case series. Br J Neurosurg 20:222-226. doi:10.1080/02688690600875507

25. Kulkarni AV, Guha A, Lozano A, Bernstein M (1998) Incidence of silent hemorrhage and delayed deterioration after stereotactic brain biopsy. J Neurosurg 89:31-35

26. Mascott CR, Sol JC, Bousquet P, Lagarrigue J, Lazorthes $Y$, Lauwers-Cances V (2006) Quantification of true in vivo application accuracy in cranial image-guided surgery: influence of mode of patient registration. Neurosurgery 59:ONS146-ONS156 discussion ONS146-156

27. Dorward NL, Alberti O, Palmer JD, Kitchen ND, Thomas DG (1999) Accuracy of true frameless stereotaxy: in vivo measurement and laboratory phantom studies. Technical note. J Neurosurg 90:160-168

28. Quinones-Hinojosa A, Ware ML, Sanai N, McDermott MW (2006) Assessment of image guided accuracy in a skull model: comparison of frameless stereotaxy techniques vs. frame-based localization. J Neurooncol 76:65-70. doi:10.1007/s11060-0052915-Z

29. Walton L, Hampshire A, Forster DM, Kemeny AA (1996) A phantom study to assess the accuracy of stereotactic localization, using T1-weighted magnetic resonance imaging with the Leksell stereotactic system. Neurosurgery 38:170-176 discussion 176178

30. Maciunas RJ, Galloway RL Jr, Latimer JW (1994) The application accuracy of stereotactic frames. Neurosurgery 35:682-694 discussion 694-685

31. Starr PA, Christine CW, Theodosopoulos PV, Lindsey N, Byrd D, Mosley A, Marks WJ Jr (2002) Implantation of deep brain stimulators into the subthalamic nucleus: technical approach and magnetic resonance imaging-verified lead locations. J Neurosurg 97:370-387

32. Helm PA, Eckel TS (1998) Accuracy of registration methods in frameless stereotaxis. Comput Aided Surg 3:51-56

33. Grunert P, Espinosa J, Busert C, Gunthner M, Filippi R, Farag S, Hopf N (2002) Stereotactic biopsies guided by an optical navigation system: technique and clinical experience. Minim Invasive Neurosurg 45:11-15. doi:10.1055/s-2002-23576 